\title{
The Transcultural Diabetes Nutrition Algorithm: A Canadian Perspective
}

\author{
Réjeanne Gougeon, ${ }^{1,2}$ John L. Sievenpiper, ${ }^{3,4}$ David Jenkins, ${ }^{5,6}$ Jean-François Yale, ${ }^{7}$ \\ Rhonda Bell, ${ }^{8}$ Jean-Pierre Després, ${ }^{9,10}$ Thomas P. P. Ransom, ${ }^{11,12}$ Kathryn Camelon, ${ }^{13}$ \\ John Dupre, ${ }^{14}$ Cyril Kendall, ${ }^{15,16,17}$ Refaat A. Hegazi, ${ }^{18}$ Albert Marchetti, ${ }^{19,20}$ \\ Osama Hamdy, ${ }^{21}$ and Jeffrey I. Mechanick ${ }^{22}$ \\ ${ }^{1}$ Crabtree Nutrition Laboratories, McGill University Health Centre/Royal Victoria Hospital, Montreal, \\ QC, Canada H3H 1 A1 \\ ${ }^{2}$ Crabtree Nutrition Laboratories, McGill University Health Centre/Royal Victoria Hospital H6.90, 687 Pine Avenue West, \\ Montreal, QC, Canada H3A 1A1 \\ ${ }^{3}$ Department of Pathology and Molecular Medicine, Faculty of Health Sciences, McMaster University, Hamilton, ON, \\ Canada L8N $3 Z 5$ \\ ${ }^{4}$ Toronto 3D Knowledge Synthesis and Clinical Trials Unit, Clinical Nutrition and Risk Factor Modification Centre, \\ St. Michael's Hospital, Toronto, ON, Canada M5C 2T2 \\ ${ }^{5}$ Department of Nutritional Sciences and Medicine, Faculty of Medicine, University of Toronto, \\ Toronto, ON, Canada M5S 3E2 \\ ${ }^{6}$ Clinical Nutrition and Risk Factor Modification Center, Division of Endocrinology and Metabolism, \\ and Li Ka Shing Knowledge Institute of St. Michael's Hospital, Toronto, ON, Canada M5C 2T2 \\ ${ }^{7}$ McGill University Health Centre/Royal Victoria Hospital, Montreal, QC, Canada H3A 1A1 \\ ${ }^{8}$ Division of Human Nutrition, Division of Agriculture, Food and Nutritional Science, and the Alberta Diabetes Institute, \\ University of Alberta, Edmonton, AB, Canada T6G 2E1 \\ ${ }^{9}$ Centre de Recherche de L'Institut Universitaire de Cardiologie et de Pneumologie de Québec, Québec, QC, Canada \\ ${ }^{10}$ Department of Kinesiology, Faculty of Medicine, Université Laval, Québec, QC, Canada G1V 4G5 \\ ${ }^{11}$ Division of Endocrinology and Metabolism, Capital Health, Halifax, NS, Canada B3H 2 Y 9 \\ ${ }^{12}$ Dalhousie University, Canada \\ ${ }^{13}$ Department of Allied Health, Clinical Nutrition, University Health Network, Toronto, \\ ON, Canada M5G 2C4 \\ ${ }^{14}$ Robarts Research, University of Western Ontario, London, ON, Canada N6A 5B7 \\ ${ }^{15}$ Clinical Nutrition and Risk Factor Modification Center, St Michael's Hospital, Toronto, ON, Canada M5S 3E2 \\ ${ }^{16}$ Department of Nutritional Sciences, Faculty of Medicine, University of Toronto, Toronto, ON, Canada \\ ${ }^{17}$ College of Pharmacy and Nutrition, University of Saskatchewan, Saskatoon, SK, Canada \\ ${ }^{18}$ Abbott Laboratories, Abbott Park, 43219, USA \\ ${ }^{19}$ Medical Education and Research Alliance (Med-ERA), New York, NY 10019, USA \\ ${ }^{20}$ Department of Preventive Medicine and Community Health, University of Medicine and Dentistry of New Jersey, \\ Newark, NJ 07101, USA \\ ${ }^{21}$ Joslin Diabetes Center, Harvard University, Boston, MA 02215, USA \\ ${ }^{22}$ Division of Endocrinology, Diabetes, and Bone Disease, Icahn School of Medicine at Mount Sinai, \\ New York, NY 10029, USA
}

Correspondence should be addressed to Réjeanne Gougeon; rejeanne.gougeon@muhc.mcgill.ca

Received 19 June 2013; Accepted 8 December 2013; Published 16 January 2014

Academic Editor: Annabel E. Barber

Copyright (C) 2014 Réjeanne Gougeon et al. This is an open access article distributed under the Creative Commons Attribution License, which permits unrestricted use, distribution, and reproduction in any medium, provided the original work is properly cited. 
The Transcultural Diabetes Nutrition Algorithm (tDNA) is a clinical tool designed to facilitate implementation of therapeutic lifestyle recommendations for people with or at risk for type 2 diabetes. Cultural adaptation of evidence-based clinical practice guidelines (CPG) recommendations is essential to address varied patient populations within and among diverse regions worldwide. The Canadian version of tDNA supports and targets behavioural changes to improve nutritional quality and to promote regular daily physical activity consistent with Canadian Diabetes Association CPG, as well as channelling the concomitant management of obesity, hypertension, dyslipidemia, and dysglycaemia in primary care. Assessing glycaemic index (GI) (the ranking of foods by effects on postprandial blood glucose levels) and glycaemic load (GL) (the product of mean GI and the total carbohydrate content of a meal) will be a central part of the Canadian tDNA and complement nutrition therapy by facilitating glycaemic control using specific food selections. This component can also enhance other metabolic interventions, such as reducing the need for antihyperglycaemic medication and improving the effectiveness of weight loss programs. This tDNA strategy will be adapted to the cultural specificities of the Canadian population and incorporated into the tDNA validation methodology.

\section{Introduction}

Type 2 diabetes (T2D) is a chronic disease with hyperglycaemia as its characteristic feature, resulting from defects in insulin secretion and/or insulin action [1]. The disorder is associated with adiposity, particularly central abdominal adiposity [2], and multiple metabolic abnormalities that increase the risk of mortality from cardiovascular diseases (CVD) by two- to fourfold [3], the leading cause of death [4], shortening life by 5 to 15 years. In Canada, the prevalence of T2D is increasing at epidemic proportions, affecting more than three million Canadians, with 6 million others at elevated risk of developing the disease. Of particular concern are non-Caucasians who comprise more than $25 \%$ of the Canadian population (Figure 1) and are highly susceptible to T2D when adopting a Western lifestyle. Diabetes affects economic prosperity, costing the Canadian healthcare system $\$ 12.2$ billion annually, a number that is projected to rise to $\$ 16.9$ billion by 2020 [4].

Canadian census data show that more than 200 tongues are spoken in Canada, 60 being aboriginal. The mother tongue reported by 6.8 million Canadians (21\% of the population) differs from English or French, the two official languages of the country. Another 4.7 million Canadians speak a language at home by order of prevalence: Punjabi, Chinese, Spanish, Italian, German, Cantonese, Tagalog, Arabic, and Mandarin (Statistic Canada). Challenged by this situation, the Canadian Diabetes Association (CDA) has begun to tailor its nutrition therapy tool, Just the Basics, to the cultural and personal tastes of individuals of varied ethnicities who have T2D (http://www.diabetes.ca/diabetesand-you/nutrition/just-basics/Accessed January 24, 2013).

Through the support of the CDA, clinical practice guidelines (CPG) for nutrition therapy [4] were published in 2003 and 2008 and updated in 2013, to provide evidence-based recommendations for healthy food choices and lifestyles that improve glycaemic, metabolic, and weight control. Included in these guidelines is the recommendation to replace highglycaemic index (GI) carbohydrate foods by low GI carbohydrate foods in mixed meals because low GI intake is associated with a lower glycaemic response and improvements in A1C [4]. A multitude of cultures and diverse geographic locations across Canada have challenged the effectiveness of nutrition therapy guidelines to promote sustained healthy eating habits in the diabetic population. In response, Mechanick et al. [5] have designed a global tDNA template for the optimization of nutritional care in prediabetes and T2D on a global scale with the intention that provided information will suit geographic and ethnocultural factors for individualization and implementation at regional and local levels worldwide. It is anticipated that tDNA will increase awareness of the benefits of dietary behaviour changes, which can be better achieved when recommended dietary patterns and food choices accommodate regional differences in genetic factors, food availability and preferences, lifestyles, and cultures. Thereafter, a task force was selected among Canadian health care experts in diabetes and nutrition to adapt the global tDNA template to Canadian mores, norms and population demographics (Figure 1). These experts, who are authors of this paper, are also key regional stakeholders in the implementation of the CDA CPG.

\section{Methods}

The process of modifying the tDNA to Canada involved a group of experts who reviewed and considered revising all of the topics outlined in the global template [5]. These reviewers also defined a vision for the Canadian tDNA and considered factors unique to the Canadian population and the guidelines and recommendations put forth by CDA in their revision, that is, ethnocultural lifestyle input; individual risk stratification with tables on classification by body composition; general recommendations on physical activity and healthy eating, with the related tables providing physical activity and nutritional guidelines; specific recommendations for obesity, hypertension, and dyslipidemia; criteria for bariatric surgery; description of an antihypertensive diet and other dietary patterns; and the glyceamic indices and load of common foods. During development of the Canadian version of tDNA, the task force established that their shared vision of the tDNA was to enable sustainable healthy lifestyle behaviours amongst healthcare providers and people with diabetes. It became evident that primary care providers would best be implicated in promoting a healthy lifestyle in their patients with T2D if they believed in its positive impact on glycaemic and metabolic control to the point that they themselves adapt and sustain a healthy lifestyle, the latter made achievable through resources and tools within the Canadian tDNA. There was also a need for defining simple assessment measures for lifestyle behaviour that put Canadians at risk of developing T2D, 


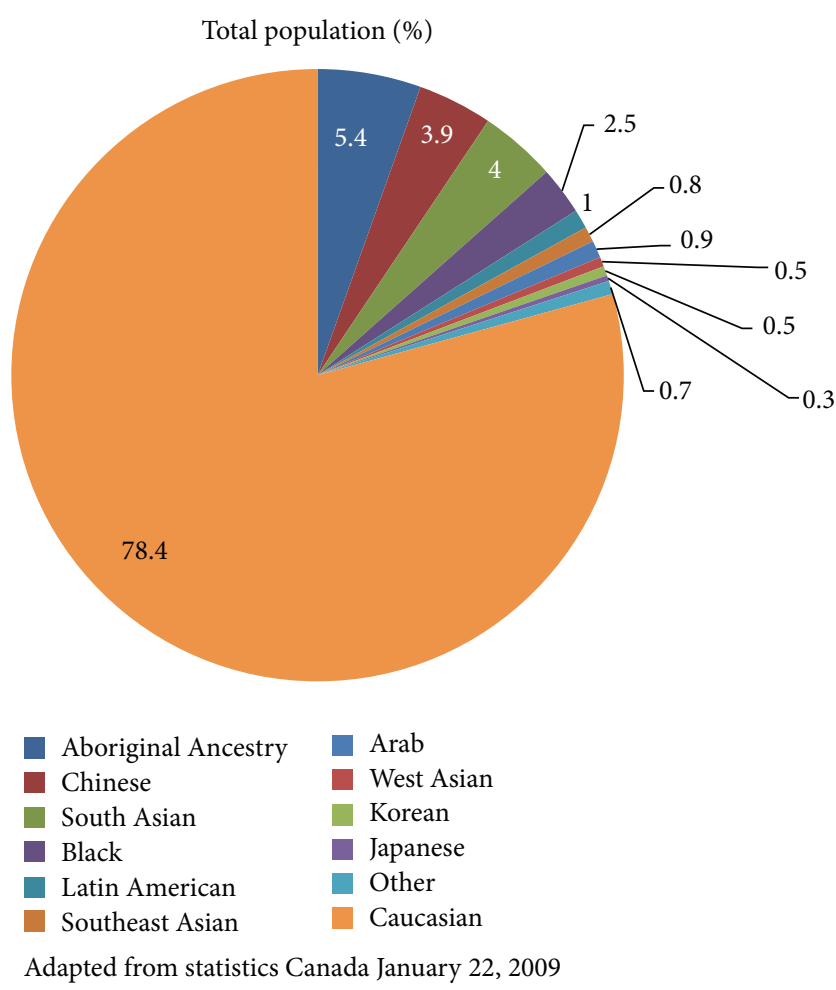

FIgURE 1: Canadian Population Demographics.

or associated complications, and also measures of lifestyle behavioural change.

Changes brought to the global tDNA template and different points of significance are highlighted in the results section of this report.

\section{Results}

As a result of our revision, the modified algorithm focused on the process of adapting healthy behaviours rather than weight loss; behaviour leading to improved diet and regular physical activity became the interventional target. Emphasis was placed also on increasing patient and provider awareness of the process of change and of improvements to lifestyle behaviour over time. The resultant objective of tDNA Canada became support of behavioural change through simple and effective dietary and physical activity advice at the primary care level. Additionally, a quick, simple, pragmatic, validated questionnaire to assess combined work and leisure time physical activity shown to be associated with mortality in a prospective population study was found to be feasible for use in clinical practice [6]. The algorithm was modified to better assist physicians in improving lifestyle habits of Canadian patients who present with diverse ethnic backgrounds, based on the existing Canadian guidelines for prevention and treatment of T2D. The global tDNA [5] adapted to fit current Canadian guidelines is presented in Figure 2.

As in the global tDNA, initially, ethnooncultural identification and geographic location are assessed concurrently with individual risk stratification, the latter described by Yusuf et al. [3]. In Canada, however, the general recommendations for counselling on care, physical activity, and healthy eating habits conform to CDA 2013 CPG [4] and are for all patients independent of the magnitude of their risk. Furthermore, the recommendations are extended to address patients' obesity, hypertension, dyslipidemia, and/or dysglycaemia with specific dietary approaches and metabolic targets. In all cases, follow-up evaluation is recommended at 1-3 months initially and at 3-6 months ongoing. The Canadian tDNA, like other cultural versions, includes and refers to tables that convey additional information adapted according to national or regional recommendations, in this case, Canadian CPGs. Examples are given in Tables 1-5.

Specific to the Canadian tDNA, Table 1 presents diabetes nutrition therapy in a manner that facilitates the selection of a strategy based on individualized targeted outcomes. Different dietary patterns evaluated in T2D populations, popular weight loss approaches, specific foods, varied macronutrient distributions, and meal replacements are listed with their specific effects on hemoglobin Alc (A1C), weight, blood pressure, lipid risk factors, inflammatory markers, hypoglycemia, and other advantages and disadvantages related to their impact on nutrients, gastrointestinal tract, or renal load. This approach is also promoted in the CDA CPGs [4].

Certain approaches from the global tDNA [5] were adapted to the many ethnicities in the Canadian population. Table 2 is an example that shows a list of common foods and their GI. Other modifications to the global tDNA were based on the recommendations from CDA CPG [4]. For example, Table 3 summarizes those for physical activity in the management of diabetes [4] and Table 4 the Dietary Approaches to Stop Hypertension (DASH) in diabetes adapted according to CDA CPG. Table 5 summarizes CDA CPG for bariatric surgery, which may be considered in patients with T2D and a BMI $\geq 35 \mathrm{~kg} / \mathrm{m}^{2}$ when lifestyle interventions have failed to achieve and maintain weight goals. Minimally invasive surgical approaches should be used by a well-established surgical team that includes experts in nutritional and psychological support. Bariatric surgery is now becoming an accepted option for the management of T2D and has been shown to be superior to medical management for its treatment [6]. Presently there is no overall consensus as to what kind of procedure is most effective, be it malabsorptive, restrictive, or combination surgery.

\section{Discussion}

Adopting healthy behaviour rather than only attaining sustained weight loss was defined as the main objective of the Canadian tDNA. This objective is consistent with CDA CPG, which recommend that self-management education incorporating knowledge and skill development, as well as cognitive behavioural interventions, should be implemented for people with diabetes (CDA CPG 2008) [4]. To optimize change, messages regarding nutrition recommendations and lifestyle modification should accommodate a person's culture [7]. The ethnic mosaic of the Canadian population provides a rich testing ground for learning how to adapt educational 


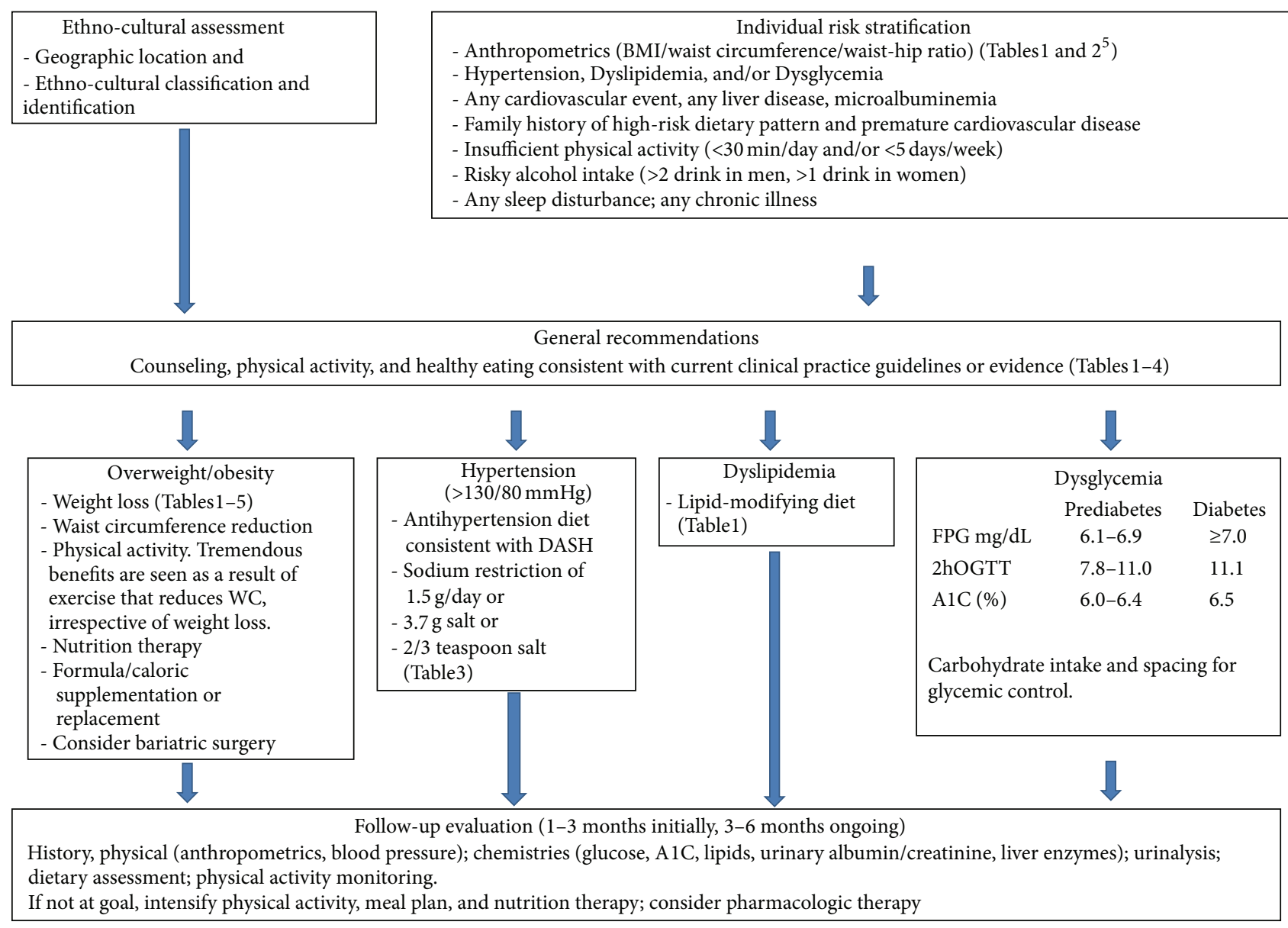

FIgure 2: Canadian Transcultural Diabetes Nutrition Algorithm (tDNA) for prediabetes and type 2 diabetes.

tools to various cultures. At this time, the dietary education tool, Just the Basics, has been created for South Asian, Latin American, and the Aboriginal communities in Canada, using a consultative process within the respective cultural groups [8]. These tools were developed by reaching out to the ethnic communities through professional and community group networks to identify persons who could contribute to the adaptation of the educational materials. People with diabetes and their family members, as well as Aboriginal, Latin American, or South Asian dietitians, other dietitians experienced in working with cultural groups, and an advisory group of dietitians with expertise in diabetes management, participated in focus group discussions and pilot-tested the tools. The focus groups explored topics such as dietary patterns practiced in Canada, meaningful expressions of portion sizes, cultural holidays and values, preferred teaching and learning methods, classification of foods into food groups, and barriers to education [9]. Just the Basics provides clear initial messages to patients about healthy eating and physical activity for diabetes prevention and management, using culturally distinct foods and languages. Although these culturally adapted tools for the South Asian, Latin American, and Aboriginal populations need ongoing assessment of their ability to promote sustained behaviour change associated with optimal diabetes control, they provide the groundwork for creating tools tailored to other high-risk populations.

The Canadian tDNA is to promote low-GI carbohydrate foods within a healthy dietary pattern. GI provides an assessment of the quality of carbohydrate-containing foods based on their effect on postprandial blood glucose [10]. To decrease the glycaemic response to dietary intake, low-GI carbohydrate foods can replace high-GI carbohydrate foods. More detailed lists can be found in the International Tables of Glycaemic Index and Glycaemic Load Values [11]. Metaanalyses of controlled dietary trials of replacing high-GI carbohydrates with low-GI carbohydrates in the context of mixed meals have shown clinically significant improvements in glycaemic control over 2 weeks to 6 months in people with type 1 diabetes (T1D) or T2D [12-14]. Replacing highGI carbohydrates with low-GI carbohydrates in mixed meals also has been shown to reduce total cholesterol over 2 to 24 weeks in people with and without diabetes [13], postprandial glycaemia and high-sensitivity C-reactive protein (hsCRP) over 1 year in people with T2D [15], and the number of hypoglycaemic events over 24 to 52 weeks in adults and children with T1D [14]. Similar benefits have been shown when low-GI diets are compared with different control diets. Dietary advice to consume a low-GI diet compared with 


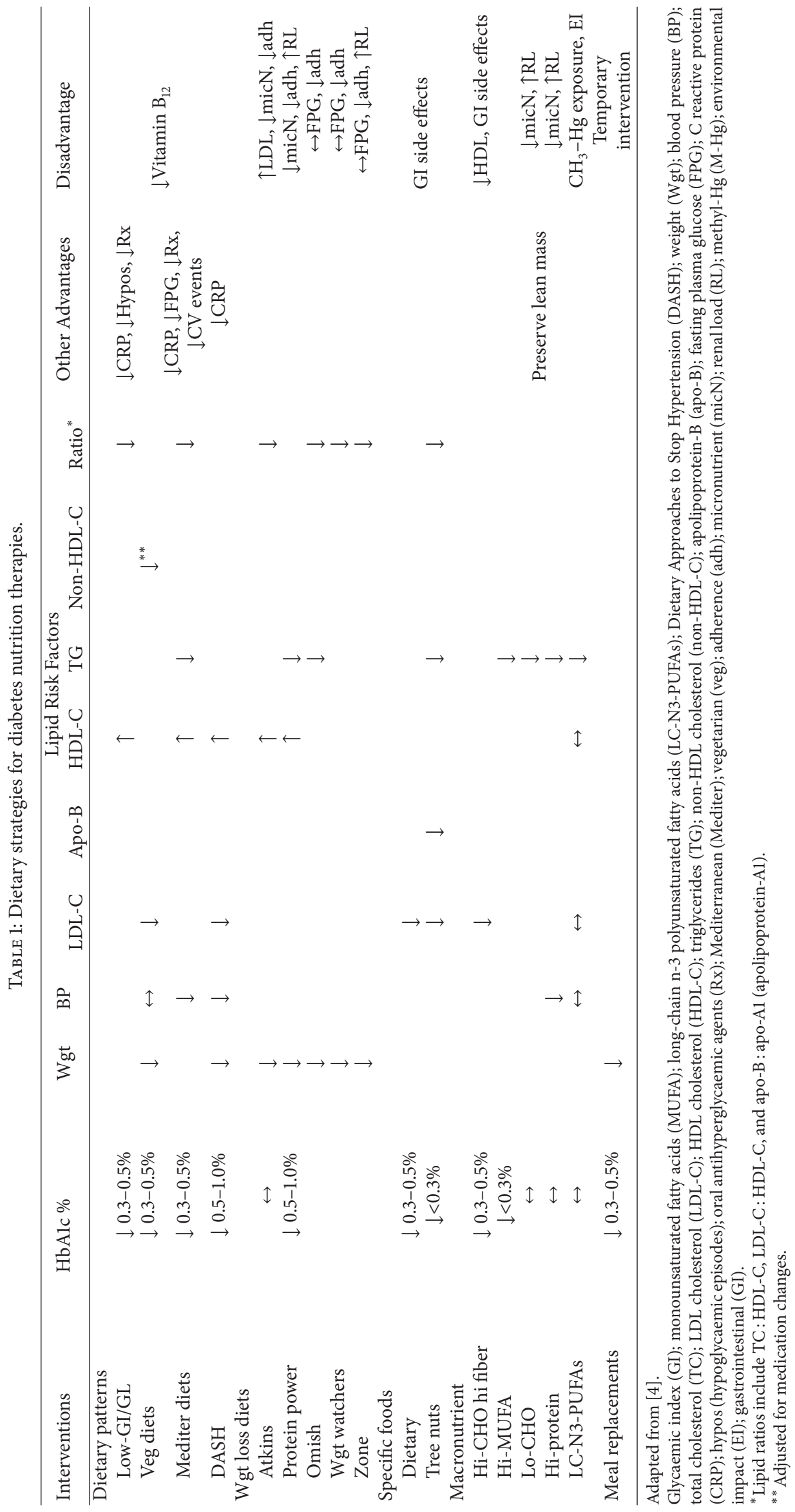


TABLE 2: Common carbohydrate foods and their glyceamic indices (GI).

\begin{tabular}{|c|c|}
\hline Food & GI \\
\hline \multicolumn{2}{|l|}{ Cereals } \\
\hline Biscuits & 69 \\
\hline Cornflakes & 81 \\
\hline Instant oatmeal & 79 \\
\hline Rice congee & 78 \\
\hline Rolled oatmeal & 55 \\
\hline Millet porridge & 67 \\
\hline Muesli & 57 \\
\hline \multicolumn{2}{|l|}{ Common items } \\
\hline Brown rice & 68 \\
\hline Barley & 28 \\
\hline Chapati & 52 \\
\hline Corn & 52 \\
\hline Corn tortilla & 46 \\
\hline Couscous & 65 \\
\hline Multigrain bread & 53 \\
\hline Rice noodles & 53 \\
\hline Spaghetti & 49 \\
\hline Udon noodles & 55 \\
\hline Wheat roti & 62 \\
\hline White rice & 73 \\
\hline White wheat bread & 75 \\
\hline Whole wheat bread & 74 \\
\hline \multicolumn{2}{|l|}{ Dairy products } \\
\hline Ice cream & 51 \\
\hline Skim milk & 37 \\
\hline Soy milk & 37 \\
\hline Rice milk & 86 \\
\hline Whole milk & 39 \\
\hline Yogurt & 41 \\
\hline \multicolumn{2}{|l|}{ Fruits } \\
\hline Apple & 36 \\
\hline Banana & 51 \\
\hline Dates & 42 \\
\hline Mango & 51 \\
\hline Orange & 43 \\
\hline Peach & 43 \\
\hline Pineapple & 59 \\
\hline Watermelon & 76 \\
\hline \multicolumn{2}{|l|}{ Legumes } \\
\hline Chickpeas & 28 \\
\hline Kidney beans & 24 \\
\hline Lentils & 32 \\
\hline Soy beans & 16 \\
\hline \multicolumn{2}{|l|}{ Snacks } \\
\hline Chocolate & 40 \\
\hline Popcorn & 65 \\
\hline Potato chips & 56 \\
\hline Rice crackers & 87 \\
\hline Soda & 59 \\
\hline
\end{tabular}

TABLE 2: Continued.

\begin{tabular}{lc}
\hline Food & GI \\
\hline Vegetables & 78 \\
Potato, boiled & 63 \\
Potato, fried & 87 \\
Potato, instant mash & 63 \\
Sweet potato & 39 \\
Carrots, boiled & 64 \\
Pumpkin, boiled & 55 \\
Plantain & 53 \\
Taro, boiled & 48 \\
Vegetable soup & \\
\hline
\end{tabular}

Glyceamic index (GI) ranks carbohydrates according to their ability to raise blood glucose levels, with the following cut-offs: low-GI $\leq 55$, medium-GI 56-69, and high-GI $\geq 70$. Adapted from Mechanick et al. [5] .

TAble 3: Canadian Diabetes Association Physical Activity Recommendations for diabetes management.

(1) Patients with diabetes should accumulate a minimum of 150 minutes of moderate-to-vigorous intensity aerobic exercise each week, spread over at least 3 days of the week, with no more than 2 consecutive days without exercise.

(2) People with diabetes (including elderly people) should also be encouraged to perform resistance exercise 3 times per week, in addition to aerobic exercise. Initial instruction and periodic supervision by an exercise specialist are recommended.

(3) An exercise ECG stress test should be considered for previously sedentary individuals with diabetes at high risk for CVD who wish to undertake exercise more vigorous than brisk walking (Grade D LOE).

Adapted from the Canadian Diabetes Association Clinical Practice Guidelines Expert Committee.

Canadian Diabetes Association 2008 clinical practice guidelines for the prevention and management of diabetes in Canada. Can J Diabetes.2008;32 (suppl 1):S1-S201.

a high-cereal fibre diet in people with T2D has been shown to improve glycaemic control and HDL cholesterol over 6 months [16]. In another trial in which dietary pulses (e.g., beans, chickpeas, lentils, and peas) were emphasized to lower the GI of the diet, significant improvements in glycaemic control and blood pressure were reported over 3 months [17]. A low-GI diet compared with a low-carbohydrate, high mono-unsaturated fat diet, has been shown to improve betacell function over one year in people with T2D [18]. Moreover, low-GI diets compared with dietary advice based on the nutrition recommendations of varied diabetes associations have been shown to have advantages. For example, (a) dietary advice to consume a low-GI diet improved glycaemic control over 3 months in Japanese people with impaired glucose tolerance (IGT) or T2D when compared with the nutritional recommendations of the Japanese Diabetes Society [19], and (b) the need for antihyperglycaemic medications over one year was decreased in people with poorly controlled T2D when compared with the nutritional recommendations of the American Diabetes Association [20]. 
TABle 4: Dietary Approaches to Stop Hypertension (DASH) for diabetes nutrition therapy.

\begin{tabular}{|c|c|c|c|c|}
\hline \multirow{2}{*}{ Food groups } & \multicolumn{3}{|c|}{ Servings per day } & \multirow{2}{*}{ Serving size } \\
\hline & $1600 \mathrm{kcal} /$ day & $2600 \mathrm{kcal} / \mathrm{day}$ & $3600 \mathrm{kcal} /$ day & \\
\hline Grains & 6 & $10-11$ & $12-13$ & $\begin{array}{l}1 \text { slice bread; } 1 \mathrm{oz} \text { dry cereal; } 1 / 2 \text { cup } \\
\text { cooked rice, pasta, cereal }\end{array}$ \\
\hline Vegetables & $3-4$ & $5-6$ & 6 & 1 cup raw leafy; $1 / 2$ cup cut raw or cooked \\
\hline Fruits & 4 & $5-6$ & 6 & $\begin{array}{l}1 \text { medium piece; } 1 / 4 \text { cup dried; } 1 / 2 \text { cup } \\
\text { fresh, frozen, canned; } 1 / 2 \text { fruit juice }\end{array}$ \\
\hline Low/nonfat dairy & $2-3$ & 3 & $3-4$ & 1 cup milk or yogurt; $1.5 \mathrm{oz}$ cheese \\
\hline Lean meat, poultry, and fish & $3-6$ & 6 & $6-9$ & $1 \mathrm{oz}$ cooked, meats, fish; 1 egg \\
\hline Nuts, seeds, and legumes & 3/week & 1 & 1 & $\begin{array}{l}1 / 3 \text { cup nuts; } 2 \text { tbsp peanut butter; } 2 \text { tbsp } \\
\text { seeds; } 1 / 2 \text { cup cooked legumes }\end{array}$ \\
\hline Fats and Oils & 2 & 3 & 4 & $\begin{array}{l}1 \text { tsp soft margarine (nonhydrogenated); } 1 \\
\text { tsp veg oil; } 1 \text { tbsp mayonnaise; } 2 \text { tbsp } \\
\text { salad dressing }\end{array}$ \\
\hline Sweets, added sugars & 0 & $\leq 2$ & $\leq 2$ & $\begin{array}{l}1 \text { tbsp sugar; } 1 \text { tbsp jelly or jam; } 1 / 2 \text { cup } \\
\text { sorbet, gelatin; } 1 \text { cup lemonade }\end{array}$ \\
\hline
\end{tabular}

Adapted from the Canadian Diabetes Association.

Canadian Diabetes Association, DASH diet summary, accessed at http://www.diabetes.ca/documents/about-diabetes/DASH_Diet_Summary.pdf on 11, 01, 2012.

TABLE 5: CDA’s Clinical Practice Guidelines Suggestions for bariatric surgery.

(1) Adults with clinically severe obesity (BMI $\geq 40 \mathrm{~kg} / \mathrm{m}^{2}$ or $\geq 35 \mathrm{~kg} / \mathrm{m}^{2}$ with severe comorbid disease) may be considered for bariatric surgery when lifestyle intervention is inadequate to achieve healthy weight goals.

(2) Bariatric surgery in adolescents should be limited to exceptional cases and performed only by experienced teams.

(3) A minimally invasive approach should be considered for weight loss surgery when an appropriately trained surgical team and appropriate resources are available in the operating theatre.

The product of mean GI and total carbohydrate intake is known as glycaemic load (GL) and has also been explored in therapeutic studies. A low GL was found to improve the efficiency of weight loss advice over 4 weeks [21] and improve risk factors for coronary heart disease including highdensity lipoprotein cholesterol (HDL-C), triglycerides, and C-reactive protein (CRP) over 4 weeks to 6 months [21-23] compared with a low-fat diet, in young overweight and obese adults without diabetes. A low GL diet has also been shown to have advantages for coronary heart disease in a systematic review and meta-analysis of prospective cohort studies [24] and for diabetes management itself in different analyses of the Nurses Health Study $[25,26]$. The success of weight loss strategies using low-GL diets appears to be related to the degree of insulin resistance as assessed by the 30-min postprandial insulin loads [23].

The Canadian tDNA integrates and emphasizes physical activity. The recommendations are based on evidence from prospective observational studies showing that individuals who perform such levels of activity have reduced risk of premature total and cardiovascular mortality as well as reduced risk of developing T2D [27-30]. The relationship between level of physical activity and mortality/morbidity is semi-independent from the concomitant influence of wellestablished CVD risk factors such as lipids, blood pressure, diabetes, and smoking [28, 31]. Thus, even among individuals who are abdominally obese with other features of the metabolic syndrome, those who reported being very active exhibit a $50 \%$ reduction in coronary risk compared to similarly matched individuals who reported being very sedentary [32]. These results show that regular physical activity not only reduces the risk of developing T2D [33, 34] but also provides clinical benefits among patients with T2D or with the features of the metabolic syndrome. Some studies have used cardiorespiratory fitness (CRF) as an objective physiological marker of participation in vigorous physical activity and have shown that a high level of CRF is associated with a substantially reduced risk of premature mortality, CVD mortality, and CVD morbidity [35-37]. The substantial cardioprotection conferred by a high level of CRF has even been reported among patients with T2D [38]. For instance, Church and colleagues [38] have shown that overweight/obese but fit patients with diabetes were at lower mortality risk than nonobese but unfit patients with diabetes. All the above observations clearly highlight the critical importance of recommending regular physical activity and better cardiorespiratory fitness in patients with T2D. In addition, regular physical activity produces substantial benefits in high-risk individuals with prediabetes, reducing their risk of converting to T2D and developing detrimental cardiovascular outcomes [33, 34].

In addition to aerobic training, moderate-to-high intensity resistance training is beneficial in order to maintain lean body mass, particularly in the aging population of patients with T2D [39-41]. As there is a dose-response relationship between level of physical activity and clinical outcomes, guidelines from the Canadian Society of Exercise Physiology 
have emphasized the greater health benefits that are expected from a greater volume of weekly physical activity [27]. In line with the Canadian recommendations, it is herein proposed to reduce the time devoted to sedentary behaviour, to increase the level of moderate-to-vigorous physical activities and exercise, and also to perform resistance exercise training for all major muscle groups. Unfortunately, accelerometer data obtained from the 2007-2009 Canadian Health Measures Survey have revealed that only about $15 \%$ of Canadian adults accumulate 150 minutes of moderate-to-vigorous physical activity per week, and this statistic is probably even worse among patients with T2D [8]. Because lifestyle modification is a cornerstone of the management of cardiometabolic risk in patients with $\mathrm{T} 2 \mathrm{D}$, it is proposed that efforts and resources should be devoted to help patients afflicted by a societal metabolic disease recalibrate their nutritional and physical activity/exercise habits.

Furthermore, interventions such as motivational interviewing, which is a specific way of helping people recognize and formulate an action plan to address specific lifestyle changes, can be useful for clients who are reluctant or ambivalent about changing behaviour [42]. The strategies used for motivational interviewing are more supportive than confrontational, and the overall goal is to increase a person's intrinsic motivation to change rather than having change imposed by healthcare practitioners [42]. Motivational interviewing, when administered by general practitioners who received training in this treatment modality, has been shown to positively affect attitudes for change in people with T2D [42].

Indeed, it must be remembered that patients find adherence to appropriate dietary patterns exceptionally difficult to maintain consistently and that recommended dietary patterns are not well followed. Furthermore, in the past, diabetes nutrition therapy has emphasized individual macronutrient and micronutrient components and their adequacy. Although studying individual nutrients may lead to an understanding of important biological mechanisms, it has been recognized more recently that providing practical advice or identifing strategies on how people eat is not sufficient. Rather, assessment of dietary patterns offers a comprehensive and complementary approach to apply nutritional principles to "real life" [43] and to identify and validate those that support optimal glycaemic control in people with T2D, regardless of extant pharmacological management. Such assessment has been suggested to be important for advancement of efficacious and effective clinical and public health interventions [43]. Analyses of food patterns would include the possibility that interactions or synergistic effects among individual foods or nutrients are examined [43].

Studies reviewed by Kris-Etherton et al. provide evidence that food-based approaches and dietary patterns reduce risk for cardiovascular disease [44]. For instance, the Breast Cancer Detection Demonstration Project [45] evaluated 42,254 women and demonstrated that all-cause mortality decreased by quartile of recommended food score. The recommended food score was the sum of the number of foods as recommended by current dietary guidelines (fruits, vegetables, whole grains, low-fat dairy and lean meats, and poultry) that were consumed. The age-adjusted relative risk for allcause mortality in persons in the upper quartile was 0.69 (95\% confidence interval 0.61-0.78); for the second and third quartiles the relative risks were 0.82 (95\% confidence interval $0.73-0.92)$ and 0.71 (95\% confidence interval $0.62-$ 0.81 ), respectively. The study demonstrated that as the quality of the dietary pattern improved (on the basis of current dietary guidelines) an associated health benefit was gained. Other reviewed [44] dietary patterns associated with lower or higher risk of chronic disease (resp.) include the Prudent Pattern compared to the Western Pattern, dietary patterns identified in the Nurse's Health Study and the Physician's Health Study. The Prudent Pattern was characterized by a higher intake of vegetables, fruits, legumes, whole grains, and fish while the Western Pattern by a higher intake of processed meat, red meat, butter, high-fat dairy products, eggs, and refined grains. Relative risk for coronary heart disease (CHD) decreased from the lowest to highest quintiles of Prudent Pattern score (relative risk 1.0 and $0.70,95 \%$ confidence interval 0.56-0.86; $P=0.0009$ for trend), whereas CHD risk increased with increasing quintile for Western Pattern score (relative risk 1.0 and 1.64, 95\% confidence interval 1.24-2.17; $P<0.0001$ for trend). These analyses may provide useful evidence for making specific food-based dietary recommendations within the context of the existing dietary guidelines; however, their impact as part of clinical treatment for diabetes has not been studied.

The Dietary Approaches to Stop Hypertension (DASH) Study demonstrated that a dietary pattern high in fruits, vegetables, and low-fat dairy products, coupled with sodium restriction, reduced hypertension and, consequently, is included here as a dietary intervention for hypertensive patients with T2D (Table 1). Moreover, the Lyon Diet Heart Study showed that dietary patterns have a marked beneficial impact on important risk factors for CVD, as well as morbidity and mortality end-points. These results support the potential positive implications for clinicians and patients of using a dietary pattern approach that emphasizes "what to eat" (i.e., plantbased foods, selected unsaturated fats) rather than "what to restrict" (i.e., total fat, saturated fat, sodium, and sugars) and give more explicit instructions that can be put into practice over the long term. Because evidence continues to emerge about the importance of regularly including particular foods (i.e., nuts, legumes, and vegetables) relative to the risk of developing diabetes and/or other chronic diseases, especially CVD, the Canadian tDNA makes reference to these items (Table 1) in its algorithm. However, background food intake patterns are not usually reported in studies in which one or two foods/nutrients are manipulated, and this information is required in order to understand the potential for interactions between foods and nutrients. Furthermore, it is unlikely that emphasizing a single food or set of foods (e.g., only low GI foods) will significantly and positively impact glycaemic control unless the changes in total intake reflect a significant change in underlying regular dietary patterns [46]. Thus, an emerging issue that must be resolved is that inclusion of particular foods is made within a diet that confers the optimal dietary pattern for risk reduction in a way that promotes 
Physician buy-in on impact of healthy behavior

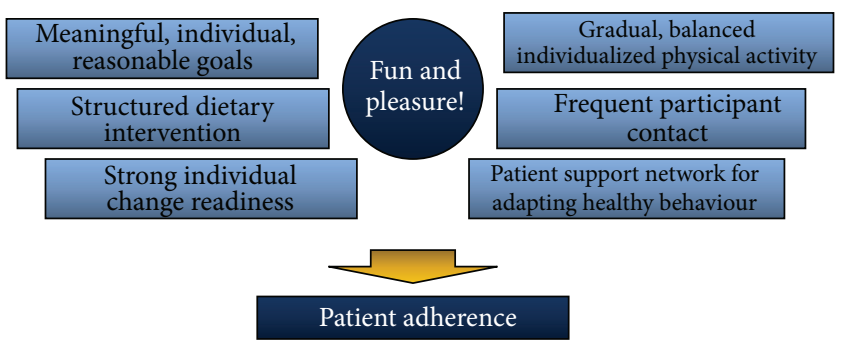

FIGURE 3: Drivers of adherence for the Canadian tDNA.

a healthy body weight (i.e., it does not exceed energy requirements).

Validated evaluation tools and simple/efficient processes for monitoring/surveillance are essential to the success of tDNA. Establishing efficacy is important, but it is also essential to identify techniques, tools, and environmental factors that contributed to, or detracted from, the success of implementing the intervention [47]. Nutrient intake, although important, does not capture the complexity of behavioural changes that people make to implement the dietary advice they received. Details are critical so that programmes can be expanded and adapted when warranted. For the field to advance, we must know what study participants were asked to do and what they actually did and we must move beyond "intention to treat" analyses. Assessment of dietary and physical activity behaviour and behaviour changes requires evaluation tools that are validated, reliable, and easy to use in various clinical practice settings [48]. The tools should be adapted to the cultural specificities of the clients and the Canadian guidelines. An inventory of validated tools, their selection according to accessibility and appropriateness, their adaptation to geographic and ethnocultural specificities, and their modification to improve clarity, simplicity, and userfriendliness remain to be achieved before validation of the tDNA is undertaken in a clinical setting. The tDNA and its tools can only be of use when patients adopt and adhere to the recommendations. This is optimized if the physicians and their patients buy-in on the impact of healthy behaviour on diabetes management and points to the importance of their involvement at each step of the tDNA. Figure 3 summarizes what is to be accomplished when primary caretakers adopt the Canadian tDNA and its tools in a clinical practice; at its core, we promote to always aim at making the relationship to food and physical activity fun and pleasurable.

\section{Conclusions}

Adapting the global tDNA template to a Canadian society led to the recognition that primary care practitioners need to participate as active and key promoters of healthy lifestyle behaviour with other members of the health professional team. Their involvement entails the development of simple, quick, and effective methods to assess nutritional and physical activity behaviours that put patients at risk and requires the implementation of strategies to help change thi behaviour in a sustainable manner. The foods in the environment of Canadians with T2D should be nutritionally adequate, culturally acceptable through appropriate food and distribution systems, physically and economically accessible at all times, and safe and secure in order to enable adoption of behaviour that promotes optimal diabetes care and make healthy food choices the norm. Furthermore, simple tools should be put in place to first evaluate sedentary behaviour and physical activity habits of patients and then support economically viable solutions to help patients increase their physical activity habits and regular exercise level. Above all, primary care practitioners should buy-in on the impact of healthy lifestyle behaviours on diabetes management by adopting this behaviour themselves. The Canadian tDNA is a first step.

\section{Disclosure}

The paper of this article was created and enriched solely by task force members through a process of ongoing literature searches, independent contributions and reviews, and group interactions for consensus.

\section{Conflict of Interests}

The authors declare that there is no conflict of interests regarding the publication of this paper.

\section{Acknowledgments}

Financial support was provided by Abbott Nutrition International. Other support may have been provided to task force members as follows. Jean-François Yale has received financial support for advisory boards, lectures, and research from Sanofi, Eli Lilly, NovoNordisk, Merck, Bristol-Myers Squibb, Astra Zeneca, Boehringer Ingelheim, Janssen, Medtronic, Abbott, Takeda, and GlaxoSmithKline. Thomas PP Ransom has received financial support for advisory boards, lectures and research from Merck \& Co., Novartis, Boehringer Ingelheim, Bristal-Myers Squibb, AstraZeneca, Eli Lilly, GlaxoSmithKline, Novo Nordisk and Sanofi-Aventis. Jean-Pierre Després declares associations with the following companies: Abbott, AstraZeneca, GlaxoSmithKline, Merck, Novartis, Pfizer Canada, Sanofi, Theratechnologies, and Torrent Pharmaceuticals. Cyril Kendall has received research grants, travel funding, consultant fees, and honoraria, or has served on the scientific advisory board for Abbott Laboratories, Advanced Food Materials Network, Agrifood and Agriculture Canada (AAFC), Almond Board of California, American Peanut Council, American Pistachio Growers, Barilla, California Strawberry Commission, Calorie Control Council, Canadian Institutes of Health Research (CIHR), Canola Council of Canada, The Coca Cola Company (investigator initiated, unrestricted), Danone, General Mills, Hain Celestial, International Tree Nut Council, Kellogg, Kraft, Loblaw Brands Ltd, Nutrition Foundation of Italy, Oldways Preservation Trust, Orafti, Paramount Farms, Peanut Institute, Pepsi-Co, Pulse Canada, Saskatchewan Pulse Growers, Solae, Sun-Maid, Tate 
\& Lyle, and Unilever. David Jenkins has served on the Scientific Advisory Board of Sanitarium Company, Agriculture and Agrifood Canada (AAFC), Canadian Agriculture Policy Institute (CAPI), Loblaw Supermarket, Herbal Life International, Nutritional Fundamental for Health, Pacific Health Laboratories, Metagenics, Bayer Consumer Care, Orafti, Dean Foods, Kellogg's, Quaker Oats, Procter \& Gamble, Coca-Cola, NuVal Griffin Hospital, Abbott, Pulse Canada, Saskatchewan Pulse Growers, and Canola Council of Canada; received honoraria for scientific advice from Sanitarium Company, Orafti, the Almond Board of California, the American Peanut Council, International Tree Nut Council Nutrition Research and Education Foundation and the Peanut Institute, Herbal Life International, Pacific Health Laboratories, Nutritional Fundamental for Health, Barilla, Metagenics, Bayer Consumer Care, Unilever Canada and Netherlands, Solae, Oldways, Kellogg's, Quaker Oats, Procter \& Gamble, Coca-Cola, NuVal Griffin Hospital, Abbott, Canola Council of Canada, Dean Foods, California Strawberry Commission, Hain Celestial, Pepsi, and Alpro Foundation; has been on the speakers panel for the Almond Board of California; received research grants from Saskatchewan Pulse Growers, the Agricultural Bioproducts Innovation Program (ABIP) through the Pulse Research Network (PURENet), Advanced Food Materials Network (AFMNet), Loblaw, Unilever, Barilla, Almond Board of California, Coca-Cola, Solae, Haine Celestial, Sanitarium Company, Orafti, International Tree Nut Council Nutrition Research and Education Foundation and the Peanut Institute, the Canola and Flax Councils of $\mathrm{Ca}$ nada, Calorie Control Council, Canadian Institutes of Health Research, Canada Foundation for Innovation, and the Ontario Research Fund; and received travel support to meetings from the Solae, Sanitarium Company, Orafti, AFMNet, Coca-Cola, The Canola and Flax Councils of Canada, Oldways Preservation Trust, Kellogg's, Quaker Oats, Griffin Hospital, Abbott Laboratories, Dean Foods, the California Strawberry Commission, American Peanut Council, Herbal Life International, Nutritional Fundamental for Health, Metagenics, Bayer Consumer Care, AAFC, CAPI, Pepsi, Almond Board of California, Unilever, Alpro Foundation, International Tree Nut Council, Barilla, Pulse Canada, and the Saskatchewan Pulse Growers. Dr Jenkins' wife is a Director of Glyceamic Index Laboratories, Toronto, Ontario, Canada. Albert Marchetti has received financial support for research and the development of educational materials in diabetes from Eli Lilly, Takeda, GlaxoSmithKline, BristolMyers Squibb, and Abbott Nutrition International. Osama Hamdy has received financial support as a Consultant from Abbott Nutrition and as a Speaker from Amylin/Eli Lilly and Abbott Nutrition. Jeffery I Mechanick has received honoraria for lectures and program development from Abbott Nutrition International.

\section{References}

[1] R. A. DeFronzo, R. C. Bonadonna, and E. Ferrannini, "Pathogenesis of NIDDM: a balanced overview," Diabetes Care, vol. 15, no. 3, pp. 318-368, 1992.
[2] J.-P. Despres, A. Nadeau, A. Tremblay et al., "Role of deep abdominal fat in the association between regional adipose tissue distributioin and glucose tolerance in obese women," Diabetes, vol. 38, no. 3, pp. 304-309, 1989.

[3] P. S. Yusuf, S. Hawken, S. Ôunpuu et al., "Effect of potentially modifiable risk factors associated with myocardial infarction in 52 countries (the INTERHEART study): case-control study," The Lancet, vol. 364, no. 9438, pp. 937-952, 2004.

[4] Committee. CDACPGE. Canadian Diabetes Association, "2008 clinical practice guidelines for the prevention and management of diabetes in Canada: self-management education," Canadian Journal of Diabetes, vol. 32, supplement 1, 2008.

[5] J. I. Mechanick, A. E. Marchetti, C. Apovian et al., "Diabetesspecific nutrition algorithm: a transcultural program to optimize diabetes and prediabetes care," Current Diabetes Reports, vol. 12, no. 2, pp. 180-194, 2012.

[6] G. Mingrone, S. Panunzi, A. de Gaetano et al., "Bariatric surgery versus conventional medical therapy for type 2 diabetes," The New England Journal of Medicine, vol. 366, no. 17, pp. 1577-1585, 2012.

[7] Committee CDACPGE, "Nutrition therapy," Canadian Journal of Diabetes, vol. 32, supplement 1, pp. S40-S45, 2008.

[8] R. C. Colley, D. Garriguet, I. Janssen, C. L. Craig, J. Clarke, and M. S. Tremblay, "Physical activity of canadian adults: accelerometer results from the 2007 to 2009 canadian health measures survey," Health Reports, vol. 22, no. 1, pp. 7-14, 2011.

[9] S. I. Mian and P. M. Brauer, "Dietary education tools for South Asians with diabetes," Canadian Journal of Dietetic Practice and Research, vol. 70, no. 1, pp. 28-35, 2009.

[10] D. J. Jenkins, T. M. Wolever, R. H. Taylor et al., "Glycemic index of foods: a physiological basis for carbohydrate exchange," The American Journal of Clinical Nutrition, vol. 34, no. 3, pp. 362366,1981 .

[11] F. S. Atkinson, K. Foster-Powell, and J. C. Brand-Miller, "International tables of glycemic index and glycemic load values: 2008," Diabetes Care, vol. 31, no. 12, pp. 2281-2283, 2008.

[12] J. Brand-Miller, S. Hayne, P. Petocz, and S. Colagiuri, "Lowglycemic index diets in the management of diabetes: a metaanalysis of randomized controlled trials," Diabetes Care, vol. 26, no. 8, pp. 2261-2267, 2003.

[13] A. M. Opperman, C. S. Venter, W. Oosthuizen, R. L. Thompson, and H. H. Vorster, "Meta-analysis of the health effects of using the glycaemic index in meal-planning," British Journal of Nutrition, vol. 92, no. 3, pp. 367-381, 2004.

[14] D. E. Thomas and E. J. Elliott, "The use of low-glycaemic index diets in diabetes control," British Journal of Nutrition, vol. 104, no. 6, pp. 797-802, 2010.

[15] T. M. S. Wolever, A. L. Gibbs, C. Mehling et al., "The Canadian Trial of Carbohydrates in Diabetes (CCD), a 1-y controlled trial of low-glycemic-index dietary carbohydrate in type 2 diabetes: no effect on glycated hemoglobin but reduction in C-reactive protein," American Journal of Clinical Nutrition, vol. 87, no. 1, pp. 114-125, 2008.

[16] D. J. A. Jenkins, C. W. C. Kendall, G. McKeown-Eyssen et al., "Effect of a low-glycemic index or a high-cereal fiber diet on type 2 diabetes: a randomized trial," Journal of the American Medical Association, vol. 300, no. 23, pp. 2742-2753, 2008.

[17] D. J. Jenkins, C. W. Kendall, L. S. Augustin et al., "Effect of legumes as part of a low glycemic index diet on glycemic control and cardiovascular risk factors in type 2 diabetes mellitus: a randomized controlled trial," Archives of Internal Medicine, vol. 22, pp. 1-8, 2012. 
[18] T. M. S. Wolever, C. Mehling, J.-L. Chiasson et al., "Low glycaemic index diet and disposition index in type 2 diabetes (the Canadian trial of Carbohydrates in Diabetes): a randomised controlled trial," Diabetologia, vol. 51, no. 9, pp. 1607-1615, 2008.

[19] Y. Amano, M. Sugiyama, J. S. Lee et al., "Glycemic indexbased nutritional education improves blood glucose control in Japanese adults: a randomized controlled trial," Diabetes Care, vol. 30, no. 7, pp. 1874-1876, 2007.

[20] Y. Ma, B. C. Olendzki, P. A. Merriam et al., "A randomized clinical trial comparing low-glycemic index versus ADA dietary education among individuals with type 2 diabetes," Nutrition, vol. 24, no. 1, pp. 45-56, 2008.

[21] C. B. Ebbeling, J. F. Swain, H. A. Feldman et al., "Effects of dietary composition on energy expenditure during weight-loss maintenance," Journal of the American Medical Association, vol. 307, no. 24, pp. 2627-2634, 2012.

[22] M. A. Pereira, J. Swain, A. B. Goldfine, N. Rifai, and D. S. Ludwig, "Effects of a low-glycemic load diet on resting energy expenditure and heart disease risk factors during weight loss," Journal of the American Medical Association, vol. 292, no. 20, pp. 2482-2490, 2004.

[23] C. B. Ebbeling, M. M. Leidig, H. A. Feldman, M. M. Lovesky, and D. S. Ludwig, "Effects of a low-glycemic load vs low-fat diet in obese young adults: a randomized trial," Journal of the American Medical Association, vol. 297, no. 19, pp. 2092-2102, 2007.

[24] A. Mirrahimi, R. J. de Souza, L. Chiavaroli et al., "Associations of glycemic index and load with coronary heart disease events: a systematic review and meta-analysis of prospective cohorts," Journal of the American Heart Association, vol. 1, Article ID e000752, 2012.

[25] J. Salmerón, J. E. Manson, M. J. Stampfer, G. A. Colditz, A. L. Wing, and W. C. Willett, "Dietary fiber, glycemic load, and risk of non-insulin-dependent diabetes mellitus in women," Journal of the American Medical Association, vol. 277, no. 6, pp. 472-477, 1997.

[26] M. B. Schulze, S. Liu, E. B. Rimm, J. E. Manson, W. C. Willett, and F. B. Hu, "Glycemic index, glycemic load, and dietary fiber intake and incidence of type 2 diabetes in younger and middleaged women," American Journal of Clinical Nutrition, vol. 80, no. 2, pp. 348-356, 2004.

[27] A. Kesäniemi, C. J. Riddoch, B. Reeder, S. N. Blair, and T. I. A. Sørensen, "Advancing the future of physical activity guidelines in Canada: an independent expert panel interpretation of the evidence," International Journal of Behavioral Nutrition and Physical Activity, vol. 7, article 41, 2010.

[28] R. S. Paffenbarger Jr., A. L. Wing, and R. T. Hyde, "Physical activity as an index of heart attack risk in college alumni," American Journal of Epidemiology, vol. 108, no. 3, pp. 161-175, 1978.

[29] R. S. Paffenbarger Jr., R. T. Hyde, A. L. Wing, and C.-C. Hsieh, "Physical activity, all-cause mortality, and longevity of college alumni," The New England Journal of Medicine, vol. 314, no. 10, pp. 605-613, 1986.

[30] M. J. LaMonte, S. N. Blair, and T. S. Church, "Physical activity and diabetes prevention," Journal of Applied Physiology, vol. 99, no. 3, pp. 1205-1213, 2005.

[31] S. Mora, N. Cook, J. E. Buring, P. M. Ridker, and I.-M. Lee, "Physical activity and reduced risk of cardiovascular events: potential mediating mechanisms," Circulation, vol. 116, no. 19, pp. 2110-2118, 2007.
[32] L. N. Broekhuizen, S. M. Boekholdt, B. J. Arsenault et al., "Physical activity, metabolic syndrome, and coronary risk: the EPIC-Norfolk prospective population study," European Journal of Cardiovascular Prevention and Rehabilitation, vol. 18, no. 2, pp. 209-217, 2011.

[33] W. C. Knowler, E. Barrett-Connor, S. E. Fowler et al., "Reduction in the incidence of type 2 diabetes with lifestyle intervention or metformin," The New England Journal of Medicine, vol. 346, no. 6, pp. 393-403, 2002.

[34] J. Tuomilehto, J. Lindström, J. G. Eriksson et al., "Prevention of type 2 diabetes mellitus by changes in lifestyle among subjects with impaired glucose tolerance," The New England Journal of Medicine, vol. 344, no. 18, pp. 1343-1350, 2001.

[35] S. N. Blair, H. W. Kohl III, R. S. Paffenbarger Jr., D. G. Clark, K. H. Cooper, and L. W. Gibbons, "Physical fitness and all-cause mortality: a prospective study of healthy men and women," Journal of the American Medical Association, vol. 262, no. 17, pp. 2395-2401, 1989.

[36] S. N. Blair, J. B. Kampert, H. W. Kohl III et al., "Influences of cardiorespiratory fitness and other precursors on cardiovascular disease and all-cause mortality in men and women," Journal of the American Medical Association, vol. 276, no. 3, pp. 205-210, 1996.

[37] R. Vigen, C. Ayers, B. Willis, L. deFina, and J. D. Berry, "Association of cardiorespiratory fitness with total, cardiovascular, and noncardiovascular mortality across 3 decades of follow-up in men and women," Circulation, vol. 5, no. 3, pp. 358-364, 2012.

[38] T. S. Church, M. J. LaMonte, C. E. Barlow, and S. N. Blair, "Cardiorespiratory fitness and body mass index as predictors of cardiovascular disease mortality among men with diabetes," Archives of Internal Medicine, vol. 165, no. 18, pp. 2114-2120, 2005.

[39] T. S. Church, S. N. Blair, S. Cocreham et al., "Effects of aerobic and resistance training on hemoglobin Alc levels in patients with type 2 diabetes: a randomized controlled trial," Journal of the American Medical Association, vol. 304, no. 20, pp. 22532262,2010

[40] R. J. Sigal and G. P. Kenny, "Combined aerobic and resistance exercise for patients with type 2 diabetes," Journal of the American Medical Association, vol. 304, no. 20, pp. 2298-2299, 2010.

[41] R. J. Sigal, G. P. Kenny, N. G. Boulé et al., "Effects of aerobic training, resistance training, or both on glycemic control in type 2 diabetes: a randomized trial," Annals of Internal Medicine, vol. 147, no. 6, pp. 357-369, 2007.

[42] S. Rubak, A. Sandbæk, T. Lauritzen, and B. Christensen, "Motivational interviewing: a systematic review and meta-analysis," British Journal of General Practice, vol. 55, no. 513, pp. 305-312, 2005.

[43] D. K. Tobias, C. Zhang, J. Chavarro et al., "Prepregnancy adherence to dietary patterns and lower risk of gestational diabetes mellitus," The American Journal of Clinical Nutrition, vol. 96, no. 2, pp. 289-295, 2012.

[44] P. M. Kris-Etherton, T. D. Etherton, J. Carlson, and C. Gardner, "Recent discoveries in inclusive food-based approaches and dietary patterns for reduction in risk for cardiovascular disease," Current Opinion in Lipidology, vol. 13, no. 4, pp. 397-407, 2002.

[45] A. K. Kant, A. Schatzkin, B. I. Graubard, and C. Schairer, "A prospective study of diet quality and mortality in women," Journal of the American Medical Association, vol. 283, no. 16, pp. 2109-2115, 2000. 
[46] S. H. Cheong, L. J. McCargar, B. W. Paty, C. Tudor-Locke, and R. C. Bell, "The first step first bite program: guidance to increase physical activity and daily intake of low-glycemic index foods," Journal of the American Dietetic Association, vol. 109, no. 8, pp. 1411-1416, 2009.

[47] R. C. Bell, "Intensive dietary advice significantly improves $\mathrm{HbA}(1 \mathrm{c})$ in people with type 2 diabetes who remain hyperglycaemic despite optimised drug treatment," Evidence-Based Nursing, vol. 14, no. 1, pp. 26-27, 2011.

[48] D. J. Toobert, S. E. Hampson, and R. E. Glasgow, "The summary of diabetes self-care activities measure: results from 7 studies and a revised scale," Diabetes Care, vol. 23, no. 7, pp. 943-950, 2000. 


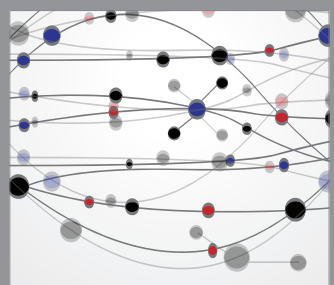

The Scientific World Journal
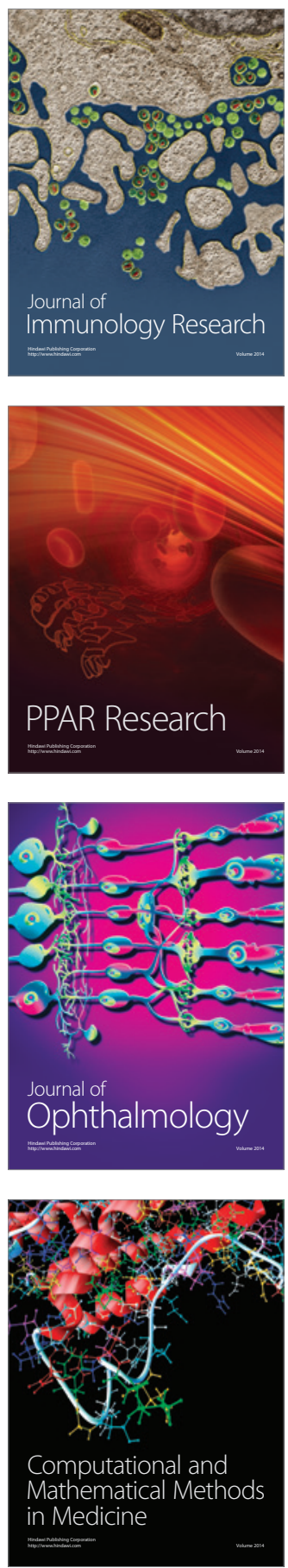

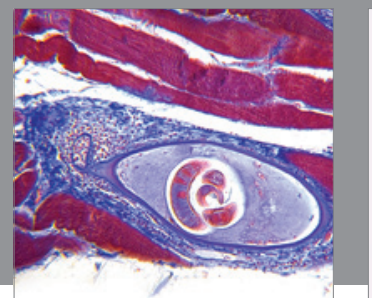

Gastroenterology

Research and Practice
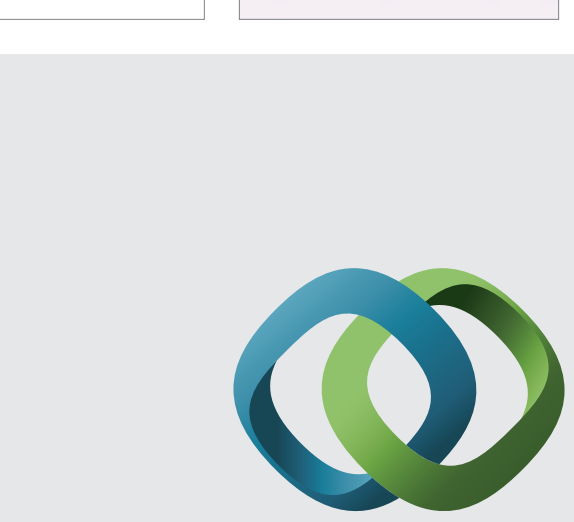

\section{Hindawi}

Submit your manuscripts at

http://www.hindawi.com
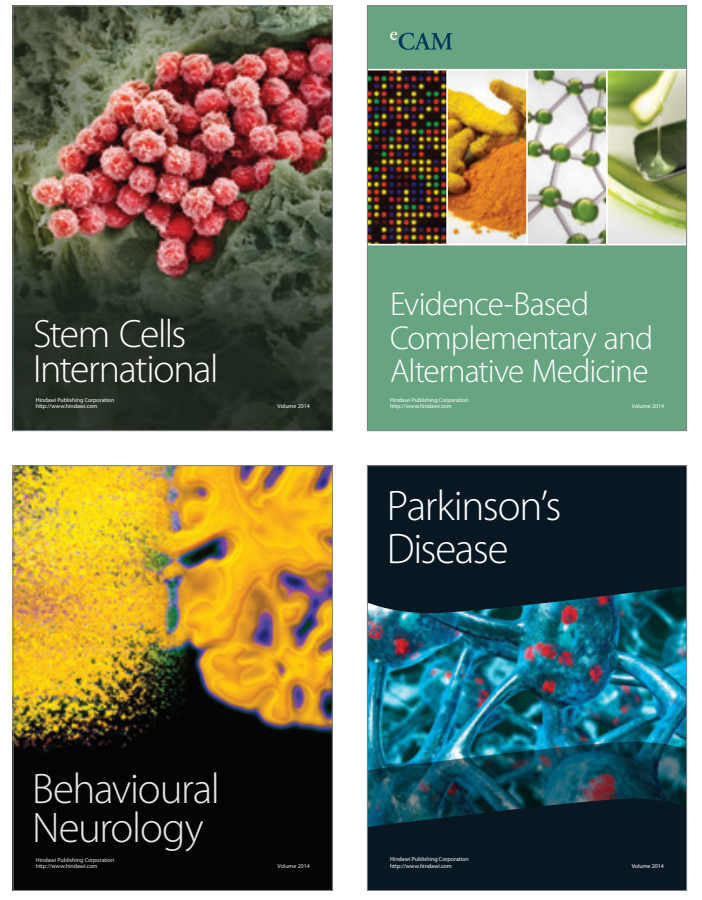
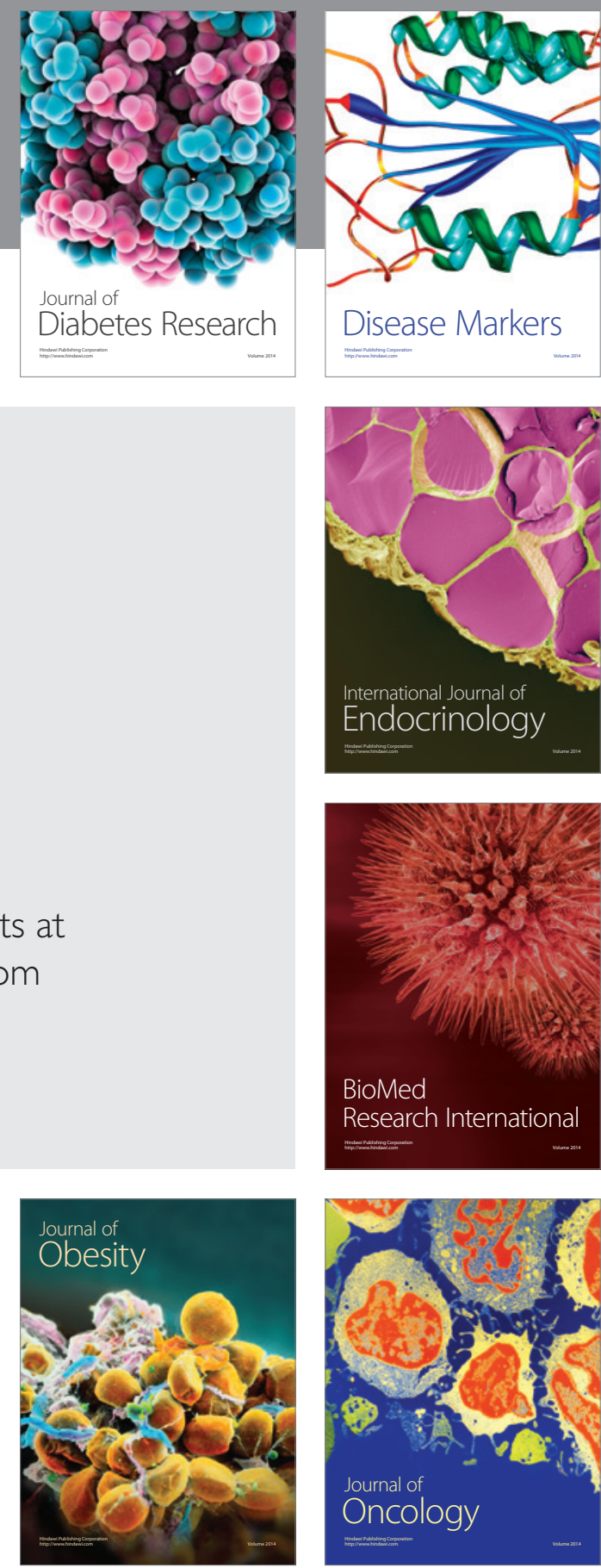

Disease Markers
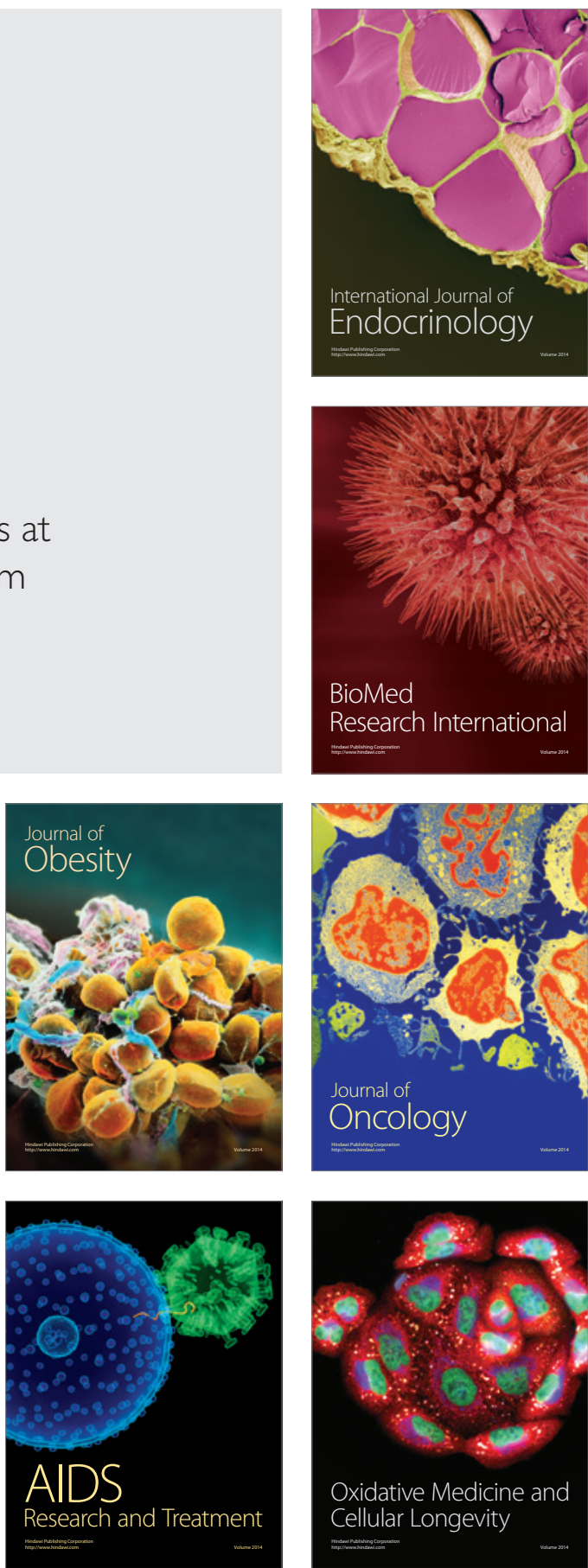